Archives de sciences sociales des religions

160 | octobre-décembre 2012

Bulletin Bibliographique

\title{
Sur les religions en Extrême-Orient
}

\section{Françoise Aubin}

\section{OpenEdition}

Journals

Édition électronique

URL : http://journals.openedition.org/assr/24268

DOI : $10.4000 /$ assr. 24268

ISSN : $1777-5825$

Éditeur

Éditions de l'EHESS

\section{Édition imprimée}

Date de publication : 30 décembre 2012

Pagination : 11-34

ISSN : 0335-5985

\section{Référence électronique}

Francoise Aubin, «Sur les religions en Extrême-Orient ». Archives de sciences sociales des religions [En ligne], 160 | octobre-décembre 2012, mis en ligne le 20 février 2016, consulté le 30 avril 2019. URL http://journals.openedition.org/assr/24268 ; DOI : 10.4000/assr.24268 


\section{Notes critiques}





\section{Françoise Aubin}

\section{Sur les religions en Extrême-Orient}

\section{Le grand Matteo Ricci, missionnaire en Chine}

À propos de :

Fontana Michela, Matteo Ricci, 1552-1610. Un jésuite à la cour des Ming. Préface de Marianne Bastid-Bruguière. Traduction de l'italien par Robert Kremer, Florence Leroy \& Ugo Lumbroso, Paris, Salvator, coll. "Biographies ", 2010, $3^{\mathrm{e}}$ édition revue, 456 p. (version originale : Matteo Ricci: Un gesuita alla corte dei Ming, Milan, Arnoldo Mondatori Ed., 2005), 450 p.

Hsia R. Po-chia, A Jesuit in the Forbidden City. Matteo Ricci, 1552-1610, Oxford, Oxford University Press, $1^{\text {re }}$ éd. 2010, $1^{\text {re }}$ éd. en paperback et réimpression avec corrections 2012, XVI, 359 p.

Deux livres à peu près simultanés sur le même personnage ? On ne peut s'empêcher de les mettre aussitôt en parallèle. La surprise commence dès l'illustration de couverture : le même portrait du jésuite à barbe frisée, en noir et blanc regardant vers la droite, du même côté que le soleil, chez Fontana ; en couleurs regardant vers la gauche chez Hsia, face opposée au soleil : l'un est sans aucun doute une copie de l'original, un portrait peint, dans le style italien, sitôt après la mort de Ricci en 1610, par un frère coadjuteur You Wenhui (connu sous le nom portugais qui lui a été donné comme à tout converti chinois de ce temps, Manuel Pereira). Ne peut-on suggérer que l'original pourrait bien être la version donnée par Hsia et conservée à l'église du Gesù à Rome ? Le personnage regarderait vers la gauche, car l'écriture chinoise se lit de gauche à droite, alors que la copie italienne qui en aurait été tirée le ferait regarder, comme il est normal en Europe, vers la droite pour lire un texte imaginaire en latinisation ${ }^{1}$.

Voyons d'abord ce qu'il faut attendre de chacun des deux ouvrages. Michela Fontana est une historienne des sciences dont le métier est de faire de la vulgarisation, dit-elle. Elle a vécu quatre ans en Chine : elle n'est, certes, pas sinologue, mais a pu bénéficier des conseils de collègues chinois. Ses sources sont principalement les collections d'œuvres en italien laissées par Ricci et ses confrères. Quant

1. Je remercie Marie Chabbert pour sa suggestion. 
à la bibliographie générale, elle est squelettique : composée de dix-sept titres en français, souvent bien loin du sujet et donnés, absurdement, par ordre chronologique à partir de leur plus récente publication. Po-chia Hsia, est, lui, un sinologue qui prend ses sources non seulement dans les fonds jésuites mais aussi dans les textes chinois. Et comme, de surcroît, la typographie de son ouvrage est plus resserrée que celle de l'ouvrage français, son contenu est infiniment plus dense et riche, malgré cent pages en moins.

Les deux travaux s'ouvrent sur les années de formation du futur jésuite à Rome, Fontana insistant sur son instruction mathématique, astronomique et scientifique ; puis les deux récits suivent parallèlement le même déroulement chronologique d'une aventure fascinante qui se lit comme un roman d'aventures. La version de Fontana continue à faire la part belle aux sciences, notamment à la traduction des éléments d'Euclide, celle de Hsia laisse une large place, bien méritée, au confrère et collègue de Ricci, Michele Ruggieri (1543-1607). En schématisant grossièrement le parcours de Ricci et de ses compagnons, on passe de l'attente de l'entrée en Chine à Macao en 1582 à l'arrivée en Chine du Sud à Zhaoqing en 1583 puis à Shaozhou en 1589, sous le costume de moines bouddhiques, et la rédaction avec Ruggieri des premiers travaux lusitano-chinois (puisque la mission de Chine était sous le padroado portugais), le déplacement plus au nord à Nanchang en 1595, sous le costume de lettrés confucéens, l'installation à Nankin en 1599 et enfin l'arrivée à Pékin en 1601.

Le travail de Fontana était, en septembre 2011, classé par le réseau des librairies de La Procure, comme une des dix meilleures ventes en Essais et Histoire, et, reconnaissons-le, c'est une lecture parfaite pour quiconque souhaite, sans connaître grand-chose à l'histoire générale du pays, s'initier aux débuts du catholicisme en Chine, à l'introduction des sciences de la Renaissance et aux immenses différences culturelles qu'a dû vaincre Ricci. Le récit est complété par un " aprèsRicci ", fait de triomphes scientifiques, puis de persécutions, par une chronologie, un glossaire et un index des noms propres. En conclusion, le cimetière jésuite de Pékin, restauré après le vandalisme de la Révolution culturelle, est « une petite oasis de paix hors du temps ".

Le travail de Hsia est, lui, une solide contribution à l'histoire Ming autant qu'à celle de la mission jésuite en Chine. Des traductions de textes chinois, une liste des caractères chinois, une riche bibliographie avec caractères chinois, un bon index, tout le désigne comme un ouvrage de référence érudit. L'épilogue porte aussi sur l'après-Ricci, mais on remarquera les chapitres précédents portant sur les paradoxes qu'a incarnés Ricci, lui qui ne pouvait trouver de vrais amis que parmi les convertis les plus zélés et qui avait voué une haine mortelle aux bouddhistes. Faut-il choisir entre ces deux livres, tous deux également bien écrits et pleins de vie ? J'avoue ne pas pouvoir m'y résoudre et trouver du nouveau en chacun d'entre eux et je ne peux que conseiller de les lire chapitre par chapitre, en commençant chaque fois par le travail de vulgarisation de Fontana et en l'approfondissant 
grâce à l'érudition de Hsia. À côté des abondantes publications suscitées par le quatrième centenaire de la mort de Ricci, je citerai une récente mise au point de Jacques Gernet, « À propos des Mathématiques chinoises » (T’oung Pao 96, 2011, p. 279-288), qui rappelle que les différences entre mathématiques chinoises et occidentales révèlent au fond des différences générales de modes de pensée.

\section{Religions et politique dans l'Extrême-Orient prémoderne (et un peu après)}
À propos de :
LAGERWEY John (dir.), Religion et société en Chine ancienne et médié- vale, Paris, Institut Ricci \& Les Éditions du Cerf, coll. « Patrimoines. Chine » et «Patrimoines. Orientalisme », 2009, 728 p.
Brotons Arnaud, Bruneton Yannick et Kouamé Nathalie (dir.), État, religion et répression en Asie. Chine, Corée, Japon, Vietnam (XIII ${ }^{e}-X X I^{e}$ siècles), Paris, Karthala, 2011, 354 p.
Bernard-Maître Henri, Humbertclaude Pierre, Prunier Maurice, Pré- sences occidentales au Japon. Du "siècle chrétien " à la réouver- ture $d u X_{X}{ }^{e}$ siècle. Édition établie et présentée par Christophe Marquet. Paris, Les Éditions du Cerf, coll. " Histoire ", 2011, 432 p. MARSONE Pierre, Wang Chongyang (1113-1170) et la fondation du Quanzhen. Ascètes taoïstes et alchimie intérieure, Paris, Collège de France - Institut des hautes études chinoises ("Mémoires de l'Institut des hautes études chinoises », vol. XI), 2010, 473 p.

Il est rare qu'un ouvrage collectif réussisse à présenter une synthèse complète où toutes les contributions s'illuminent harmonieusement l'une l'autre autour d'un thème commun. Religion et société en Chine ancienne et médiévale est de ceux-là ; et l'exploit est d'autant plus remarquable qu'il concerne un sujet protéiforme, essentiel et pourtant mal connu : l'origine de la religion ou des religions en Chine. L'introduction de l'éditeur du volume, John Lagerwey, contribue largement au sentiment d'unité que ne peut manquer d'éprouver le lecteur, soit-il un sinologue averti ou un amateur peu éclairé, l'un et l'autre y trouvant son profit. Lagerwey en effet livre en introduction une reconstitution chronologique, où les conclusions de chacun des présents collaborateurs pour chaque sous-époque sont reliées à des travaux précédents, souvent déjà classiques. La trame en est faite, d'une façon générale, de continuité et de ruptures, et plus précisément du remplacement du dieu suprême, l'antique Shangdi, par le Ciel à l'époque Zhou, une véritable révolution religieuse.

Huit contributions illustrent l'évolution de la pensée et des pratiques religieuses dans la Chine antique. Les abondantes découvertes archéologiques de ces dernières décennies permettent à Alain Thote (p. 47-76) de mieux cerner : 
(1) les coutumes funéraires des Shang (ca. 1500 à 1045 av. J.-C., les premiers témoignages écrits datant du milieu de la dynastie), qui, héritées du néolithique, accentuent la stratification sociale, surtout dans la phase finale dite d'Anyang, alors que le roi fait visiblement le lien entre le monde des vivants et celui des morts et que les sacrifices humains rehaussent le statut du titulaire de la tombe ; et (2) celles des Zhou occidentaux ( $c a .1045$ à 771 av. J.-C.) qui reflètent un remaniement social, notamment l'indépendance grandissante des princes, un changement dans les mœurs - ainsi les vases rituels ne sont plus destinés à des boissons mais à une nourriture solide de viandes et céréales, et une diversité locale - dans la civilisation de Chu, le mobilier funéraire doit contribuer au bienêtre du défunt, désormais individualisé.

Olivier Venture (p. 77-100) étudie, durant le même temps qu'A. Thote, la pratique de la "pyro-ostéomancie ", c'est-à-dire d'une "méthode de divination consistant à brûler un support en os jusqu'à l'apparition de craquelures... ensuite interprétées comme des signes » (p. 77). Les plus anciens témoignages remontent à la seconde moitié du IV e millénaire av. J.-C. Une innovation des devins Shang a été la pyro-chéloniomancie, plus coûteuse, pour laquelle le support est le plastron ou partie ventrale d'une carapace de tortue. La pratique mantique est rationalisée et dotée d'inscriptions sous les Shang à partir du long règne du roi Wuding (ca. 1250-1192 av. J.-C.), afin de connaître les dispositions des esprits et de les apaiser. Sous les Zhou occidentaux, la scapulomancie régresse au profit de la chéloniomancie et de la nouvelle technique de l'achilléomancie et des séries de chiffres.

Marc Kalinowski (p. 101-164) poursuit le propos de Venture en analysant, avec de nombreux exemples, les pratiques mantiques à l'époque suivante, celle des Zhou orientaux (770-256 av. J.-C.), ou plus précisément celle des Printemps et Automnes, chunqiu, de 770 à 481, puis celle des Royaumes combattants de 480 à 221 av. J.-C. Des principautés de plus en plus étendues se consolident durant la première de ces deux époques pour devenir des royaumes indépendants à l'étape suivante, tandis que s'affirme un humanisme fondé sur le perfectionnement moral et la recherche de la place de l'homme dans la nature. Les techniques divinatoires ne visent plus à se concilier les dieux et les ancêtres, mais à prédire l'avenir. Elles impliquent plus souvent des scribes et des conseillers que des devins et, si elles portent encore sur l'écaille de tortue et l'achillée pour répondre à des questions concrètes, elles s'étendent à l'interprétation des présages et des rêves. Les registres divinatoires et sacrificiels du royaume méridional de Chu sont particulièrement intéressants.

Jean Lévi (p. 165-239) décèle une structuration politico-cosmique du pouvoir et une hiérarchisation par l'intermédiaire du sacrifice sanglant : depuis les Zhou au moins, gouverner c'est sacrifier, c'est-à-dire manger de la viande sacrificielle et en distribuer les restes aux inférieurs. Le noble est celui qui possède des ancêtres auxquels sacrifier, et plus il peut en revendiquer, plus exaltée est sa position. 
Quant aux grands sacrifices au Ciel et à la Terre, ils sont l'apanage du souverain. D'autre part, à l'époque des Royaumes combattants, le Dao perd toute base concrète pour devenir "une entité divine en même temps qu'un pur néant, l'expression même du non-être », et cette supériorité du non-être sur l'être va fonder l'absolutisme de Qin et des Han (p. 236).

Catherine Despeux (p. 241-275) montre l'importance, à l'époque des Royaumes combattants (453 à 222 av. J.-C.) et surtout des premières dynasties impériales Qin (221 à 206 av. J.-C.) et Han (206 av. J.-C. à 220 apr. J.-C.), des diverses pratiques de culture corporelle visant à une longévité ou une immortalité ouverte à tous. C'est d'abord la gymnastique, «technique apotropaïque, thérapeutique, prophylactique ou gériatrique » (p. 254-255) associée à la maîtrise du souffle. Ce sont aussi des pratiques diététiques et alchimiques, dont la base est l'abstinence de céréales, qui, liées à la mort, doivent être remplacées par des plantes ou des minéraux afin de rendre le corps léger. Il faut également une gestion de la sexualité. Et, pour les lettrés, une éthique morale l'emporte même sur les pratiques corporelles.

S'il est un phénomène religieux dont on parle peu lorsqu'il s'agit de la Chine, c'est bien le chamanisme. Rémi Mathieu (p. 277-304) en cerne ici les caractéristiques qui y sont assimilables, de la fin des Zhou au début des Han, soit environ du $V^{e}$ au $I^{\text {er }}$ siècle av. J.-C., de telle sorte qu'on ne pourra désormais plus guère en nier l'existence. L'équivalent du chamane est le (ou la) $w u$, qui a, selon les zones culturelles, les époques et les milieux sociaux, des formes d'action très diverses : il (ou elle) peut être "invocateur, exorciste, devin, guérisseur, faiseur de pluie "; son panthéon éventuel est multiple et le rapport qui l'unit aux esprits passe par le chant, la danse, la transe. Sous l'impulsion de la culture écrite des lettrés, les pratiques des $w u$, définies par leur oralité, ont été officiellement interdites par un édit de 32 av. J.-C., et se sont alors ancrées définitivement et jusqu'à nos jours dans les milieux populaires.

Les souverains des Qin et des premiers Han cherchaient principalement à s'approprier les différents cultes en usage à travers le pays. Comme le narre Marianne Bujard (p.305-337), la stratégie des lettrés consista, en l'espèce, à faire instaurer en 32 av. J.-C. un grand rituel réservé à l'empereur et célébré à la capitale en l'honneur de Taiyi, qui va devenir peu à peu le Ciel - alter ego de l'empereur dans le monde divin, tandis qu'étaient supprimés des centaines de cultes locaux. Mais les réformateurs ne purent éviter qu'un culte à la terre Houtu ne soit associé au Ciel et qu'un certain nombre de cultes locaux ne reçoive le soutien de la Cour, le culte aux Cinq Pics notamment et le culte aux Immortels, fussent-ils d'obédience confucéenne ou taoïste.

Un article novateur, par Michèle Pirazzoli-t'Serstevens (p. 340-393 et 31 illustr.), fait le tour d'une question essentielle, le traitement de la mort et des morts au début de l'époque impériale, sous les Qin et les Han, et remet en cause bien des idées reçues. Une transformation radicale dans la structure des tombes 
en ce temps signale, en dépit de nombreuses variations locales, des transformations sociales générales. Ainsi, la tombe, désormais horizontale, est devenue un lieu où vivants et morts communiquent et qui est destiné à assurer le confort du défunt. Sa décoration et son mobilier se rattachent à un système cosmogonique qui atteint le faîte de sa formalisation au II siècle apr. J.-C. Mais si la culture des tombes est alors extrêmement typée, la vision de l'au-delà est hétérogène, en fonction de différences socioculturelles sur lesquelles on ne saurait trop insister. Aussi l'iconographie sépulcrale n'est-elle pas facile à déchiffrer avec certitude, d'autant que les images semblent fréquemment manquer d'un lien avec le texte les accompagnant. D’une façon générale, l'univers est conçu comme une structure tripartite, dominée par les esprits du Ciel et les divinités naturelles, le monde humain étant au milieu et celui des morts, reflétant souvent l'administration impériale, sous terre ; l'on peut aussi y trouver un quatrième niveau entre Ciel et hommes, celui des immortels. Au II siècle apr. J.-C., l'iconographie funéraire, associée aux croyances en l'au-delà, comprend nécessairement Xiwangmu, «la Reine Mère de l'Occident » et des immortels, dont l'iconographie est constituée d'un mélange d'éléments autochtones et d'éléments empruntés (comme les ailes).

Huit articles encore forment la seconde partie qui couvre la «période de division ", de 221 à 589. Le directeur du projet, John Lagerwey, évoque, en trois contributions, d'abord sous l'angle des rapports entre politique et religion au cours de la vingtaine de dynasties qui se sont succédé de-ci de-là, la montée du bouddhisme et l'affirmation bien structurée de l'Église taoïste des Maîtres célestes (p. 397-428) ; puis la formation d'une littérature taoïste prolifique (p. 459-492); enfin les parties constituantes du rituel taoïste en ce temps et ses réformes (p. 565600). Les autres contributions sont consacrées au bouddhisme : par Sylvie Hureau, les traductions et, en symbiose, les apocryphes (p. 429-458); et, d'autre part, le rituel, notamment le jeûne (p. 493-529); par Françoise Wang-Toutain, la doctrine, entre spéculation métaphysique et dévotion et le rôle qu'y joua l'influence taoïste (p. 601-641); par François Martin, les idéaux et les pratiques qui se diffusèrent chez les laïcs bouddhistes (p. 531-563). Enfin, en point d'orgue, les pratiques, qui ont sans doute mutuellement interagi dans le taoïsme - la pratique du souffle notamment - et dans le bouddhisme - la visualisation et la contemplation de l'esprit (p. 643-683).

Chaque contribution jouit de sa propre bibliographie, mais un glossaire commun, qui donne tous les caractères chinois, et un bon index général font de cet ensemble remarquable un instrument d'une réflexion féconde sur la nature de la religion en Chine.

État, religion et répression en Asie offre sept dossiers de persécutions religieuses très diverses, classées chronologiquement à travers l'Asie orientale sinisée. L’introduction générale de Nathalie Kouamé (p. 7-25) justifie le choix du cadre politico-géographique, centré sur le modèle chinois, et celle du thème des antagonismes et des répressions, qui va à l'encontre de notre vision populaire 
d'un bouddhisme et d'un confucianisme iréniques. L'histoire s'ouvre sur le Japon de l'époque de Kamakura, au tout début du XIII ${ }^{\mathrm{e}}$ siècle lors de la naissance d'un régime féodal, avec le bannissement du moine Hônen coupable, au regard des écoles bouddhiques concurrentes, d'avoir prôné la pratique exclusive d'une récitation (nembutsu) du nom du bouddha Amida : c'est là un exemple d'une instrumentalisation de la violence étatique (Arnaud Broton, p. 29-71). L'exemple d'une répression religieuse totale est fourni par la Corée (dynastie des Chosŏn) au début du XV $\mathrm{XV}^{\mathrm{e}}$ siècle, durant les dix-neuf ans du règne de T’aejong (1400-1418) : une politique d'éradication de la vie monastique et des rituels bouddhiques se fit alors avec grande violence au nom de la morale confucéenne ( " néo-confucianiste », comme nous disons) et se poursuivit tout au long de la dynastie jusqu'en 1897 (Yannick Bruneton, p. 73-147). Mais certains cas de répression peuvent avoir une signification ambiguë qui appelle à leur réévaluation. Ainsi l'on peut constater que les mesures antichrétiennes prises par Toyotomi Hideyoshi, le conquérant du pouvoir dans le Japon du XVI ${ }^{\mathrm{e}}$ siècle (édit d'expulsion des missionnaires, tous des Jésuites, en 1587 et martyre de vingt-six chrétiens à Nagasaki en 1597) n'ont pas été soutenues par une politique répressive cohérente ; car, dans les faits, l'intolérance religieuse n'était pas le moteur d'action du général comme l'étaient l'affirmation de son pouvoir sur un Japon miné par la désunion et la guerre civile, et ses tentatives hégémoniques sur l'Asie orientale (Nathalie Kouamé, p. 149-182; sur les aléas du christianisme au Japon à partir de ses débuts au $\mathrm{XVI}^{\mathrm{e}}$ siècle, voir ma chronique sur "Le catholicisme au Japon. Note critique » dans la RHE, Revue d'histoire ecclésiastique, vol. 107, 2012, no 1, p. 328-339).

Un exemple de "répression endémique ", jouant de la menace et de temps à autre d'une destruction théâtralisée de lieux de culte, est fourni par la politique d'interdiction des cultes dits "immoraux » (yin) au sein du pluralisme religieux de la Chine des Qing, entre 1644 et 1898. De telles destructions ne prennent leur sens que replacées dans le contexte des rapports entre fonctionnaires et société locale et l'échec des compromis possibles (Vincent Goossaert, p. 183-221). Le caractère fluide et complexe que peuvent assumer les répressions religieuses est illustré dans le Japon de l'après-Meiji (entre 1868 et 1945) par la persécution des " nouvelles religions ", tels les mouvements Ōmoto, Tenrikyō ou Renmonkyō. Le cadre en est la restauration de l'autorité impériale d'origine divine en 1868 et l'entrée dans une modernité de type occidental et ses suites. La question qui se pose est, comme dans toute autre forme de répression religieuse, celle de déceler au nom de quoi et à quelle fin un pouvoir politique opprime-t-il une religion et évolue-t-il dans son traitement de telle ou telle minorité religieuse (Jean-Pierre Berton, p. 223-262). Un appel au cas vietnamien de la répression du caodaïsme, sous la forme précise de sa branche de Tây Ninh (près de la frontière cambodgienne) et de ses pratiques médiumniques, depuis l'arrivée au pouvoir du parti communiste en 1975, illustre l'importance du symbolisme dans l'interprétation du processus répressif : ainsi l'on voit ici à l'œuvre un usage 
politique des statistiques ou encore la récente folklorisation touristique d'une pratique religieuse officiellement jugée déviante (Jérémy Jammes, p. 263-310). Enfin le débat s'élargit en y incluant les États-Unis, lesquels sont fondés, comme la Corée et le Japon, sur la conviction d'un destin exceptionnel d'origine divine. Or, l'on constate que les États-Unis, depuis leur indépendance en 1783 et leur conviction d'être la Nouvelle Jérusalem, le Japon, à partir du début de Meiji en 1868 et de la restauration d'un pouvoir impérial d'ascendance divine, la Corée du Sud, depuis sa fondation en 1948 et son apparence de pays chrétien modelé sur l'Amérique, ont établi des frontières symboliques, dont la violation a justifié à plusieurs reprises l'accusation d'un sectarisme condamnable à l'égard notamment de dénominations protestantes. Ainsi en a-t-il été, à certains moments et dans certaines circonstances, des Mormons, des Adventistes du Septième jour, des Témoins de Jéhovah du côté américain, de l'Église de l'Unification du révérend Moon chez les Coréens et autre part dans le monde, des mouvements Tenrikyô, Ômoto et surtout Sôka Gakkai du côté japonais. Le dilemme a été, comme dans l'ensemble des pays démocratiques, de concilier la liberté religieuse et la sécurité publique menacée, entre autres, par l'ultranationalisme (Nathalie Luca, p. 311-339).

Cette passionnante collection de cas est bien organisée pour satisfaire les besoins du lecteur cherchant à s'informer honnêtement autant que du spécialiste : tableau chronologique général in fine et petits tableaux chronologiques et statistiques ponctuels au cours des articles; pour les cinq premières contributions, des listes très complètes des caractères chinois nécessaires (on regrettera cependant que J.-P. Berthon ne donne sa bibliographie japonaise qu'en latinisation). Quant à l'article traitant du Vietnam, par Jérémy Jammes, il fournit une liste des transcriptions scientifiques des noms et termes vietnamiens. Une petite critique cependant: pourquoi n'y a-t-il pas de renvoi interne lorsque les mêmes faits apparaissent dans deux contributions successives (ainsi des mouvements sectaires japonais sont traités par N. Luca et J.-P. Berthon sans que les coauteurs semblent le remarquer).

Avec Présences occidentales au Japon, un recueil d'articles rares, car parus au Japon ou en Chine entre 1937 et 1941 (et même inédits pour deux d'entre eux, les chapitres II et III de la $2^{\mathrm{e}}$ partie), nous retournons dans le Japon des ouvertures et des répressions successives dont il vient d'être fait mention. Disons tout de suite que le troisième coauteur, Maurice Prunier (un professeur de français à Tôkyô dans les années 1920 et 1930, mort en 1944), ne figure que pour une brève contribution inédite sur les fumie, des représentations chrétiennes que les chrétiens persécutés devaient fouler au pied en marque d'apostasie du XVII siècle à 1858 ( $1^{\text {re }}$ partie, $10^{\mathrm{e}}$ article du chap. III, p. 212-215, ill. 6/1 à 6/4). Quant aux deux autres auteurs, ils reçoivent en introduction (p. 11-18) une biographie insistant sur leur engagement au Japon. Au P. Pierre Humbertclaude (1899-1984), un prêtre marianiste qui fut là-bas en poste de 1931 à 1952, reviennent deux précieux articles sur la littérature autochtone à "l'âge d'or » de la christianisation $\mathrm{au} \mathrm{XVI} \mathrm{e}^{\mathrm{e}}$ siècle, telle qu'on pouvait la reconstituer à la fin des années trente. 
Les écrits religieux japonais comportaient des ouvrages doctrinaux simples à l'usage des fidèles et, pour les prêtres, des textes doctrinaux et ascétiques ; s'y ajoutait une littérature profane, qui comprenait essentiellement des monogatari, récits ayant pour thème, en l'occurrence, l'arrivée des Portugais et composés par un converti devenu frère jésuite, et des dictionnaires et grammaires rédigés en toute hâte (chap. I de la $2^{\mathrm{e}}$ partie, p. 221-272). Un aspect de l'édition catholique au Japon a été l'opposition aux jésuites que manifestèrent les missionnaires des ordres mendiants, surtout s'ils avaient été formés aux Philippines : tel est le cas du dominicain espagnol Diego Collado, une figure haute en couleurs, qui tenta en 1630 de faire mettre à l'Index à Romeun manuel jésuite, publié en 1607 sur la base de traductions de l'italien et de méditations, le Spiritual Shugyô no Manual (chap. II de la $2^{\mathrm{e}}$ partie, un inédit, p. 272-286).

Au P. Humbertclaude encore, l'on doit deux articles sur les rapports de la France avec le Japon. D'abord, une réflexion sur la présence de la France au XVIII ${ }^{\mathrm{e}}$ siècle et dans la première moitié du XIX, avant la réforme de Meiji, qui s'ouvre sur une belle bibliographie d'une cinquantaine de titres en français et néerlandais et d'un nombre un peu plus élevé de titres en japonais (donnés en écriture japonaise, en latinisation et en traduction); suit un passionnant récit de la connaissance que les Japonais ont pu avoir de la France - en l'absence de relations directes - de sa politique internationale, de son gouvernement (une large place étant faite à Napoléon), de ses régions, de ses villes et de ses monuments au cours des décennies concernées. L'information était au XVIII ${ }^{\mathrm{e}}$ siècle seulement en néerlandais : l'étude du français ne commença que dans la première décennie du $\mathrm{XIX}^{\mathrm{e}}$ siècle, par l'intermédiaire du néerlandais. De telle sorte que des fragments de la littérature française de loisir ou scientifique furent connus grâce à des traductions hollandaises, ce qui, incidemment, renseigne sur l'importance de l'influence culturelle française en Hollande au XVIII ${ }^{\mathrm{e}}$ siècle (chap. II de la $3^{\mathrm{e}}$ partie, p. 359-406). Cependant quelques Français ou Belges francophones sont connus pour avoir séjourné au Japon au XVII ${ }^{\text {e }}$ siècle ou, tout au moins, tenté d'y aller. Le plus remarquable est Paul Caron (ca.1600-1673), qui, arrivé tout jeune comme marmiton sur un navire hollandais, apprit si bien le japonais qu'il le parlait, dit-on, mieux qu'aucun Hollandais ; après vingt-deux ans de séjour sur place et la rédaction en néerlandais d'une Description du Japon, il se hissa très haut dans l'échelle sociale en dépit de multiples hostilités, finissant directeur de la Compagnie française des Indes ; ses fils, protestants, quittèrent la France lors de la révocation de l'édit de Nantes en 1685 ; néanmoins, il semble que l'écrivain Caron de Beaumarchais soit un de ses descendants. Un seul Français est mort martyr, en 1637, le dominicain Guillaume Courtet. Un chanoine d'Aix, Honoré Bonfils, lui ne trouva pas, en 1645-1647, de bateau qui veuille le prendre pour le passage vers l'Extrême-Orient, car la navigation était aux mains des Portugais et des Espagnols qui n'acceptaient que leurs compatriotes. Vers 1873, un soldat, certainement protestant, Jean de Lacombe, séjourna quelque temps dans le pays et en 
tira une description restée inédite. Par contre, l'attribution aux jésuites Faure et Bonnet d'un séjour en 1711 est le résultat d'une erreur (chap. III de la $3^{\mathrm{e}}$ partie, p. 407-423).

Le reste du recueil Présences occidentales au Japon est formé des articles et conférences du père jésuite Henri Bernard, dit Bernard-Maître (1889-1975), un grand spécialiste des missions jésuites dans la Chine du XVI ${ }^{\mathrm{e}}$ siècle. Dans le registre de la construction de l'idée que la France s'est faite du Japon, l'auteur traite, en un style alerte, de la question à l'époque de la Renaissance, entre 1545 - date d'une lettre de Saint François Xavier (bien que l'apôtre n'ait débarqué à Kyûshu qu'en 1549, marquant là la fondation du christianisme au Japon) - et 1619 départ d'un véritable antichristianisme nippon. À une bibliographie chronologique des sources disponibles en français en ce temps succède un passionnant inventaire de leur contenu : le Japon, bien que fort lointain (six années étaient nécessaires pour un aller et retour), est, somme toute, un pays riant, proche du nôtre. Mais la persécution et la proscription des missionnaires européens vont lui aliéner les sympathies qui se tourneront vers la Chine (chap. I de la $3^{\mathrm{e}}$ partie, p. 31-358). En complément au survol de ce que nous connaissons de la littérature chrétienne au Japon du XVI ${ }^{\mathrm{e}}$ siècle par le P. Humbertclaude, le présent auteur traite, d'une part, des intermédiaires chinois de la fin des Ming au déclin des Qing (chap. III de la $2^{\mathrm{e}}$ partie, p. 287-296) et, d'autre part, de la connaissance que les Japonais ont pu avoir d'ouvrages européens par le biais de traductions chinoises qui ont pénétré au Japon durant la période de fermeture de 1614 à 1853 (chap. IV de la $2^{\mathrm{e}}$ partie, p. 297-320) : deux chapitres intéressants pour les historiens de la pensée chinoise influencée par l'Occident.

Et surtout, le P. Bernard-Maître remplit toute une première partie consacrée à plusieurs aspects du siècle chrétien au Japon : le début des relations diplomatiques entre le Japon et les Espagnols des îles Philippines entre 1571 et 1594, qui tourne l'attention d'une manière très bienvenue vers les missionnaires des ordres mendiants, dominicains et franciscains (chap. I, p. 21-63); les premiers contacts du Japon avec la culture européenne, par l'intermédiaire des Portugais, des Anglais et des Hollandais en rivalité, enfin des réfugiés chinois fuyant, à la fin des Ming, l'invasion mandchoue (chap. II, p. 65-119). Enfin neuf conférences, formant presque tout le chapitre III (p. 121-217), cernent divers témoignages d'infiltrations « occidentales » avant la réouverture du pays et d'échecs : le confucianisme, cause, dit l'auteur, de la fermeture du Japon ; la mentalité commerçante et dynamique de Sakai, dans la région de l'actuelle Ôsaka ; l'influence du bouddhisme chinois à Kyôto ; l'espoir d'un épiscopat au Japon dans les années 1580-1581; l'expansion japonaise dans les mers du Sud qui permit le maintien d'un certain lien avec le monde extérieur ; l'obstacle au rayonnement européen qu'ont été les disputes entre jésuites et ordres mendiants et la rivalité entre le Portugal et l'Espagne ; le rôle des marchands autochtones ; les conséquences des deux zones de juridiction et de trafic commercial, aux frontières incertaines, entre Lisbonne 
et la voie des Indes, et Madrid et sa base en Nouvelle-Espagne (le Mexique); le scandale culturel et politique qu'a constitué pour les témoins autochtones et surtout les gouvernants le phénomène kirishitan - les chrétiens prêts au martyre pour leur foi et, en réponse, l'exaltation de la tradition nationale japonaise.

Bien des points soulignés avec brio et érudition par les PP. Humbertclaude et Bernard-Maître étaient originaux lors de leur exposition. Or ils n'ont rien perdu de leur fraîcheur. Il faut féliciter l'initiateur et éditeur de ce bel ouvrage, Christophe Marquet, un japonisant spécialiste de l'histoire du livre au Japon.

Si l'on en revient aux religions médiévales, l'étude fouillée que Pierre Marsone consacre à Wang Chongyang (1113-1170) et la fondation du Quanzhen. Ascètes taoïstes et alchimie intérieure est à remarquer. Il faut savoir que le Quanzhen ( "Achèvement de l'authenticité » selon la traduction du terme la plus plausible), initié vers 1167 en Chine du Nord, est devenu une mouvance taoïste très populaire en Chine et politiquement active jusqu'à nos jours : il est fascinant de cerner comment un courant religieux tel que celui-ci a pu se créer à partir d'une conversion personnelle, se développer en un réseau lâche de communautés monastiques et finalement prospérer en quelques décennies jusqu'à l'emporter sur les options religieuses concomitantes. Vincent Goossaert et Stephen Eskildsen avaient auparavant exposé les pratiques communautaires et institutionnelles du mouvement à ses débuts (pour le premier en une thèse doctorale défendue en 1997 et un article dans Journal of Chinese Religions, 29, 2001, p. 111-138; pour le second en un livre de 2004). L'auteur, lui, part de ce qu'il distingue comme tantôt historique tantôt légendaire dans les biographies du fondateur et de ses sept grands disciples, les " Authentiques ", et de leurs œuvres littéraires pour montrer la variété de leurs origines sociales, de leurs personnalités morales et de leurs parcours spirituels. Par-delà l'hagiographie, un examen sérieux des sources montre que le fondateur historique du mouvement, Wang Chongyang (ou Wang Zhe, 1113-1170), en réaction à une vie précédemment dissipée, se soumit à une ascèse totale à partir de 1159 et voulut dès lors convertir les habitants du Shanxi, sa région natale, puis du Shandong. Ses successeurs immédiats, «des mendiants exaltés » dit l'auteur (p. 115), en quête de légitimité, célébrèrent le rôle des Immortels dans la révélation qu'il en aurait reçue et sa propre position comme immortel transmetteur de la doctrine. Mais, de fait, la structuration du mouvement est, dans un premier temps, l'œuvre de ses disciples directs, bien que ceux-ci aient été dépourvus d'intronisations régulières.

En premier lieu, Ma Danyang (ou Ma Yu, 1123-1184), qui, lent à se convertir, fut le disciple le plus proche du maître et la figure dominante des quinze premières années du Quanzhen; on peut lui attribuer un extrême respect de la vie animale, une dévotion à son maître et une infatigable activité missionnaire, outre qu'il a été crédité deux cents ans après sa mort de talents médicaux. Resté dans l'ombre, Tan Changzhen (Tan Chuduan, 1123-1185) prêchait le détachement total de la vie matérielle, associant bouddhisme et taoïsme dans un apostolat 
populaire. Liu Changshen (Liu Chuxuan, 1147-1203) a représenté la tendance lettrée du mouvement naissant. Et surtout le célèbre Qiu Changchun (Qiu Chuji, 1148-1227), connu pour avoir conseillé Gengis-khan en 1222-1223 et avoir mené le Quanzhen à son apogée par son institutionnalisation, fut un poète fécond célébrant la paix et la beauté de la nature et auquel fut bien plus tard attribué la création de la branche majeure du mouvement, le Longmen.

À ces quatre grands disciples, la tradition a ajouté les noms de deux autres missionnaires actifs. Wang Yuyang (Wang Chuyi, 1142-1217) joua un rôle essentiel, bien que souvent méconnu, dans la formation de la doctrine et des institutions du Quanzhen. Enfin Hao Taigu (Hao Daotong, 1140-1213), devin et adepte convaincu de l'érémitisme, compléta une liste des Sept Authentiques commençant avec Wang Chongyang. Mais dans le courant du XIII ${ }^{\mathrm{e}}$ siècle et surtout à partir du XIV ${ }^{\mathrm{e}}$, afin de rendre justice aux nombreuses femmes entrées dans le mouvement - un tiers du clergé, dit-on (p. 184) - on y adjoignit le nom d'une femme, Sun Bu'er (Sun Qingjing, 1119-1183), l'épouse de Ma Danyang. De la sorte, la liste des Sept Authentiques était désormais complète, Wang étant hors liste comme fondateur.

La seconde partie du volume est d'abord consacrée à la doctrine initiale du Quanzhen principalement telle que prêchée par Wang et la théologie de celui-ci d'après ses œuvres. On notera les modèles bouddhiques et taoïstes de renoncement au monde et l'unité de pensée avec le confucianisme et le bouddhisme, ce qui peut expliquer en partie la rapidité du succès populaire du mouvement. L'adepte se reconnaît par son habit et son équipement, par sa pratique de la mendicité et de la mortification, par l'interversion de son sommeil - veille de nuit et repos le jour -, par l'égalitarisme de sa vie communautaire. Psychologiquement, la joie et la poésie tiennent une grande place dans sa vie intellectuelle. La lignée spirituelle revendiquée par Wang compte un des Immortels les plus célèbres, Lü Dongbin (p. 266-272), ainsi que Zhongli Quan et Liu Haichan. Le dernier chapitre reprend le thème de l'alchimie intérieure, neidan, déjà bien traité par ailleurs (bibliographie des études occidentales p. 287) ; mais il est évoqué ici sous son aspect ascétique et mystique plutôt qu'en pratiques physiques et psychologiques, étant entendu que l'essentiel reposait, dans la doctrine du Quanzhen, sur la paix du cœur (p. 198).

Cet ouvrage est un modèle du genre : agréable à lire pour le non-initié, grâce à l'extrême clarté de l'exposé et à la multiplicité des citations, il est aussi un bon instrument de travail, riche en caractères chinois, en références des sources originelles et des travaux chinois et japonais, complété par une chronologie efficace et un bon index. Deux critiques, mineures certes, me viennent cependant à l'esprit. D'une part, le travail est clos sur lui-même, comme si le Quanzhen n'avait jamais été traité auparavant dans une langue occidentale, les auteurs occidentaux n'étant cités que très épisodiquement pour une mauvaise lecture de caractère ou 
une petite erreur d'interprétation : il est indéniable que la biographie de Wang n'avait pas encore été abordée avec une telle amplitude; mais l'on aimerait savoir au passage quels points étaient déjà connus. D'autre part, l'affirmation que « le Quanzhen, convoité par toutes les cours, devint l'un des principaux points d'appui des Mongols pour leur conquête de la Chine» (p. 191) est contestable dans sa généralité. Les points d'appui des Mongols dans leur conquête de la Chine du Nord d'abord, puis de la Chine des Song du Sud, ont été les shibou chinois, sortes de condottieri commandant des milices locales, ralliés aux conquérants dès 1214, dans la conviction que de deux maux, le désordre ou le joug mongol, il leur fallait choisir le moindre et que le plus efficace était d'attirer le généralissime Muqali dans le camp prochinois.

\section{Religions et politique en Extrême-Orient aux $x x^{e}-x x l^{e}$ siècles}

À propos de :

Goossaert Vincent \& Palmer David A., The Religious Question in Modern China, Chicago, The University of Chicago Press, 2011, $\mathrm{XII}+464 \mathrm{p.}^{2}$

ELISSEEFF Danielle, Hybrides chinois. La quête de tous les possibles, Paris, Hazan et Musée du Louvre, 2011, 214 p., 45 ill. en couleurs.

Roux Pierre-Emmanuel, La croix, la baleine et le canon, La France face à la Corée au milieu du XIX ${ }^{e}$ siècle. Paris, Les Éditions du Cerf, 2012, 460 p.

Et qu'est donc devenu le Quanzhen à l'époque contemporaine, après les décennies de répression du régime populaire, ou encore le taoïsme en général ou les autres manifestations religieuses? Une évidente explosion des phénomènes religieux depuis une trentaine d'années a suscité, en réponse, un flot de publications occidentales exposant l'actuel succès de tel ou tel courant - chrétien le plus souvent - ou une histoire des religions en Chine au tournant du XXI siècle destinée avant tout aux non-initiés. Et voici qu'arrive The Religious Question in Modern China, dû à Vincent Goossaert et David Palmer, connus comme spécialistes, le premier du taö̈sme, le second du Falungong, et l'ouvrage s'annonce dès sa sortie comme un succès de librairie : tout de suite connu des spécialistes sous le sigle de $R Q M C$, il a bénéficié rapidement de recensions enthousiastes dans quantité de périodiques d'études extrême-orientales et même, faveur rarissime sinon unique pour un travail de sinologie n'impliquant pas le monde anglosaxon, d'un compte rendu d'une pleine page et demie dans le Times Literary Supplement (TLS, 6 avril 2012). Voyons donc ce qu'il apporte de nouveau sur un sujet que l'on aurait pu croire rebattu.

2. Traduction française : La question religieuse en Chine, Paris, CNRS Éditions, $400 \mathrm{p}$. Parution le 15/11/2012. 
C'est avant tout une étude totale qui intègre en un récit unifié tout ce qui a été dit précédemment sur les religions en Chine (y compris par les deux présents coauteurs) depuis la tentative de réforme de 1898 jusqu'à l'année 2008, terminus ad quem. Contrairement à l'accent mis habituellement sur les ruptures, l'analyse insiste ici sur la continuité des structures et des mentalités, et le résultat est plus d'une fois déroutant. Si l'on commence par évaluer le système des observances et croyances religieuses vers la fin de l'ancien régime, on lui voit un « équilibre dynamique " (p. 19) qui s'étendait à toutes les classes de la société et à tous les milieux et pénétrait tous les aspects de la culture, à travers une foule de temples, d'associations dévotionnelles, de fêtes et de pèlerinages. Le cadre institutionnel en était les "Trois Enseignements » (sanjiao) - bouddhisme, taoïsme, confucianisme, coexistant sans syncrétisme, définis chacun par son clergé, sa littérature canonique, sa liturgie, ses centres de formation. L'empereur, qui décidait de l'orthodoxie ou du rejet comme illicites des cultes et des pratiques, menait en fait un projet utopique d'implantation de normes morales communes à tous. Mais dans les dernières années des Qing, les intellectuels sapèrent cet équilibre afin de " civiliser " (jiaohua) les masses, les uns par le fondamentalisme confucéen, les autres par un discours anticlérical rejetant les religions constituées (chap. I).

Six chapitres étudient ensuite sous divers angles le développement du nouveau concept de "problème religieux », créé sur le modèle terminologique japonais et censé être responsable des échecs politiques et militaires de la Chine : ils suivent à la fois des étapes chronologiques et un découpage thématique. De 1898 à la chute de l'empire en 1911, l'action politique encouragea la transformation des temples en écoles ou leur destruction; et se développa peu à peu, à l'intérieur du discours religieux des élites, le concept de lutte contre les "superstitions » (mixin, encore un terme venu d'un néologisme japonais). Durant la période suivante de 1912 à 1927, dénommée ici étrangement et sans explication «le Beiyang » (p. 58) ou "le régime Beiyang " (p. 71) [Beiyang : litt. "Mer du Nord ", c'està-dire les provinces maritimes du Nord - Shandong, Hebei, Liaoning, par allusion aux élites militaires originaires de ces régions et qui dominaient la politique à Pékin en ce temps], cinq religions furent reconnues dans la théorie officielle le catholicisme romain, le protestantisme, l'islam, enfin des versions purifiées du bouddhisme et du taoïsme. Quant au bouddhisme tibéto-mongol et à l'islam, ils allaient désormais, et jusqu'à nos jours, être traités comme des spécificités ethniques relevant de la politique des «nationalités» (minzu, terme japonais). Le régime nationaliste tenta d'appliquer, à partir de 1927, une idéologie utopique basée sur la science et l'éradication des héritages néfastes du passé, mais en vain (chap. II).

Simultanément aux actions gouvernementales, les leaders religieux s'efforçaient de développer la conception d'une religion modèle, championne de la modernité. Les élites protestantes, bien éduquées dans les établissements fondés par les missionnaires anglo-saxons, donnèrent le ton, en envisageant favorablement jusque dans les années 1920 la création d'une république chrétienne et en 
appuyant constamment la notion d'une citoyenneté religieuse par laquelle les pieux adeptes devaient servir d'importateurs des valeurs civiques en usage en Occident et surtout en Amérique du Nord, tout en défendant avec honnêteté les intérêts nationaux. Ce modèle normatif chrétien influença plusieurs courants réformistes bouddhistes (notamment le bouddhisme humaniste de Taixu et l'engagement matériel des moines dans la guerre sino-japonaise des années 1930 et 1940). Son effet se manifesta aussi, mais avec un succès plus limité, dans une tradition taoïste réinventée que les leaders du Quanzhen essayèrent de dominer. Le confucianisme de son côté s'inventa une tradition de prosélytisme missionnaire et un culte de Confucius digne de rivaliser avec une liturgie chrétienne. Un des mérites du présent ouvrage est d'inclure dans sa rétrospective l'islam de langue chinoise : une éducation réformée, grâce à une meilleure connaissance de l'arabe (et, ajoutonsle, de la littérature islamique en chinois classique mise en termes néoconfucéens) ainsi qu'un engagement de citoyen responsable et patriotique ont caractérisé l'islam chinois à l'époque de la République et durant la guerre sino-japonaise (chap. III).

Mais l'histoire des politiques gouvernementales et des mouvements de réformisme enclenchés par les leaders des cinq religions ne couvre pas la totalité du champ religieux aux époques considérées, loin de là. L'emprise du triangle intellectuel formé par les nouveaux concepts de science/religion/superstition s'est étendue aussi à la pratique locale, et, tout d'abord à l'occasion de l'extraordinaire effervescence culturelle des quinze premières années de la République, à la myriade d'associations et d'assemblées qui quadrillaient à la base le monde des croyants. Ont fleuri alors une multitude d'associations salvatrices ou spiritualistes qui synthétisaient des tendances spirituelles traditionnelles et qui, par le nombre de leurs adeptes, l'emportaient largement sur les mouvements réformistes des cinq religions officielles (le mouvement Cao Dai, fondé en 1926 au Vietnam, en est un avatar, p. 102). Puis, à l'époque du Parti nationaliste à partir de 1927, ce fut au contraire la mise en valeur, comme représentatif de "l'essence nationale ", d'un élément particulier des techniques traditionnelles de soin au corps et à l'esprit, ainsi la médecine traditionnelle, les arts martiaux et le qi gong qui seront institutionnalisés par le régime communiste en 1950 (chap. IV).

Cependant les discours modernistes ne pénétrèrent guère dans les campagnes où les suppressions de temples, les destructions de statues, la confiscation de terres à destination religieuse et la répression des "superstitions " rencontrèrent des oppositions, plus ou moins graves selon les lieux et les moments. Dans les cas extrêmes, ce furent des rébellions armées et des mouvements millénaristes, rationalisés comme des "sociétés secrètes " (encore un terme venu du japonais vers 1912, lui-même traduit de l'anglais) par les observateurs extérieurs qui n'en comprenaient pas la logique religieuse. Le processus de répressions et résistances entraîna une configuration nouvelle du paysage religieux, les sociétés salvationistes occupant tout le champ laissé libre, surtout en Chine du Nord, ainsi le Yiguandao 
issu d'un mouvement existant depuis le début du XVIII siècle et développé dans les années 1930 (chap. V).

Le parti communiste (PCC), fondé en 1921 par des intellectuels nourris de l'idéal des Lumières, adhéra à ses débuts à la théorie de la " Nouvelle Culture ", le mouvement défendu par le Kuomintang (KMT), tout en critiquant le recours à la violence. L'expérience de la Longue Marche de 1934 à 1936, qui l'avait mis en contact avec des populations frontalières, Yi, Tibétains, musulmans, conduisit Mao Zedong à renoncer à son idéologie antireligieuse dans ses rapports avec elles depuis sa base de Yan'an; et, sous le couvert de la diplomatie du Front uni antijaponais à partir de 1937, il rechercha l'alliance de toute espèce d'association et institution religieuse, lesquelles étaient aussi victimes d'infiltrations par les Japonais. La reprise de la lutte entre le PCC et le KMT après la reddition du Japon en 1945 entraîna des scissions à l'intérieur de nombreuses communautés à coloration religieuse, selon qu'elles suivaient l'un ou l'autre parti. Mais lorsque le régime communiste fut en voie d'être vainqueur sur le continent, il commença à éliminer ce qu'il appelait maintenant des "sectes " et des " sociétés secrètes ", c'est-à-dire les sociétés salvatrices jugées contre-révolutionnaires. Cependant les nouvelles formes d'organisation du travail rural se fixèrent souvent autour des groupes de parenté patrilinéaire, lesquels purent survivre ainsi jusqu'à nos jours bien que privés de leur vie rituelle précédente. Enfin le nouveau régime procéda, à partir de 1947 déjà, à une institutionnalisation de ses rapports avec les cinq grandes religions tout en continuant à accorder son soutien aux traditions sécularisées telles que la médecine traditionnelle ou les arts martiaux, jusqu'à ce que cet équilibre soit remis en question par la campagne antidroitière et le Grand bond en avant de 1958-1961, puis tragiquement par la Révolution culturelle (chap. VI).

Nous atteignons ici le cœur de la démonstration des deux auteurs et leur irremplaçable apport à l'histoire chinoise moderne : contrairement à ce qu'on imagine, la Révolution culturelle ne représente pas un mouvement de sécularisation, mais « l'apothéose d'un courant parallèle de sacralisation politique qui prend ses racines dans la culture chinoise politique et religieuse de l'empire, autant que dans les dimensions utopiques et apocalyptiques de la révolution moderniste » (p. 167). C'est ainsi que se décèlent quatre niveaux dans une religiosité politique, messianique et utopique, implantée graduellement tout au cours du $\mathrm{XX}^{\mathrm{e}}$ siècle : les rituels civiques propres à tout État-nation; la moralisation de la vie civile et politique, promue particulièrement par le mouvement de la Vie nouvelle du KMT dans les années 1930 et celui de la Renaissance culturelle à Taiwan dans les années 1970 ainsi que dans la "réforme de la pensée » à Yan'an; dès les années 1930, l'ascétisme révolutionnaire destiné à créer une race de futurs martyrs pour la cause du communisme; enfin le culte de Mao et ses suites (chap. VII).

Un inventaire des multiples modernités religieuses à l'entrée du XXI siècle occupe les six chapitres suivants, formant une seconde partie d'actualité, après 
la première partie historique. Une qualité supplémentaire du présent ouvrage est sa capacité à prendre en compte les solutions adoptées à travers l'ensemble de la diaspora chinoise, à Taiwan bien sûr, d'abord en position de colonie hollandaise puis japonaise, enfin après la prise brutale de pouvoir par le KMT en 1945, au Manchukuo sous tutelle japonaise de 1931 à 1945, à Singapour, à Hong Kong, en Asie du Sud-Est, etc. Les traditions religieuses chinoises y ont survécu, plus ou moins modifiées, longtemps considérées par les milieux intellectuels comme une marque d'arriération, mais estimées de nos jours comme le signe d'une authenticité chère aux touristes ; puis voici que depuis les années 1980 elles reviennent vers la Chine populaire (chap. VIII).

La valeur la plus importante dans la culture chinoise, et la plus intraitable à travers les changements de normes, est sans contredit la "piété filiale » (xiao) dont l'aspect essentiel est le culte rendu aux ancêtres défunts : belles funérailles dans un lieu dont la géomancie est favorable et nourrissement de l'âme par des offrandes quotidiennes, ce sont des obligations que les républicains ont associées à un confucianisme malfaisant et ont voulu remplacer par des rites occidentaux. Le régime communiste tenta, d'une manière plus radicale encore, d'imposer l'incinération du défunt et l'organisation d'un simple service commémoratif par son unité de travail. La relaxation politique depuis les années 1980 a permis, en milieu rural, un retour à l'ensevelissement dans un terrain personnel et, chez soi, l'établissement d'un petit autel domestique consacré aux ancêtres avec des photographies remplaçant l'ancienne tablette funéraire. Mais dans les grandes villes, où ne subsistait plus de support pour une perpétuation des actes religieux, ce sont des supermarchés funéraires qui prennent en charge les funérailles (chap. IX).

Si l'on quitte la sphère intrafamiliale pour considérer les phénomènes religieux sous leur aspect communautaire depuis les années 1980, l'on constate à Taiwan et en milieu villageois continental un extraordinaire foisonnement de reconstructions et restaurations de temples et de fêtes religieuses, fonctionnant sur fonds privés, donc dans une logique d'économie marchande et de succès touristique, avec une liturgie gravement appauvrie (chap. X).

En Chine continentale, les villes ayant été, dans les années 1990 et 2000, entièrement reconstruites, sans que des lieux de culte aient été prévus par les planificateurs, alors qu'il ne reste debout que quelques temples et mosquées gérés par les associations officielles des cinq religions à titre de musées et d'attraction touristique, les citadins manquent de repères dans leurs traditions religieuses. Halloween, "Christmas ", Santa Claus et les thérapies New Age font un malheur dans les milieux des jeunes branchés. Pourtant toute culture religieuse n'a pas disparu, en partie grâce au souvenir de concepts et de croyances maintenu par les femmes. Dans les villes, se sont organisées de nouvelles formes de pratiques religieuses officiellement acceptables, bien qu'elles échappent aux institutions orthodoxes. Ce sont d'abord les arts martiaux, popularisés par les films de kung-fu, des bandes dessinées, souvent japonaises, et à partir du milieu des années 1990 
par internet depuis les pays de diaspora chinoise ; le végétarisme comme pratique morale justifiée par un modèle occidental; tous les mouvements de culture du corps, dont le qi gong et le falungong ont été les meilleures expressions à l'époque de Deng Xiaoping. À Taiwan, après la levée de la loi martiale en 1987, et en Asie du Sud-Est, les sociétés salvatrices inspirées du Yiguandao et de sa popularisation du confucianisme ont fourni une idéologie aux classes ascendantes et ont envahi le champ religieux de la Chine populaire après la proscription du mouvement qi gong en 1999. Le bouddhisme humaniste laïque, prôné par Taixu, a donné naissance à Taiwan à la Fondation Tzu Chi (Ciji), maintenant principale ONG de l'île et pôle de l'adhésion bouddhique en ce lieu. Enfin, la poussée remarquable du christianisme en Chine continentale est due au succès des mouvements évangéliques protestants et du culte dans des églises domestiques, "House Church Movement » (une petite méprise a été relevée p. 1 : ce n'est pas le nombre total des chrétiens qui se serait multiplié par soixante depuis 1949, mais peut-être celui des protestants et évangéliques seulement, quoique p. 287 une expansion démographique protestante chiffrée vers l'an 2000 à trente fois semble davantage plausible). On peut de la sorte parler d'une culture religieuse urbaine et « moderne » dans le monde chinois, ayant pour caractéristiques d'être spontanée et individuelle et de se présenter comme héritière d'une tradition viscéralement chinoise (chap. XI).

Quel a été le cadre officiel dans lequel les différents courants de la religiosité rurale et urbaine postmaoïste ont pu se développer ? Tandis que l'atmosphère politique se détendait depuis la fin des années 1970, les autorités modifiaient leur discours pour souligner, de plus en plus au cours du temps, les aspects positifs des religions, notamment dans les domaines de la morale et de la philanthropie. Mais elles gardaient le monopole de la définition du légal institutionnalisé et de l'illégal réprimé (par exemple au cours de la croisade contre « la pollution spirituelle» de 1982-1984). Le système de gestion gouvernementale des religions institutionnalisées, aboli par la Révolution culturelle, fut remis en vigueur en 1978-1980. Ce sont, au niveau central, le Front uni, rattaché au Parti ; le Bureau des Affaires religieuses devenu en 1998 l'Administration d'État des Affaires religieuses, dépendant de l'État; les associations religieuses officielles dites «patriotiques » représentant les cinq grandes religions ; enfin des académies et des séminaires pour la formation du clergé et des intellectuels. Mais l'application de la législation en la matière (notamment le « document 19 » de mars 1982, le «document 6 » de février 1991 et la réouverture au culte des monuments religieux accaparés par un tourisme financièrement rentable) dépend en pratique des instances provinciales et locales, ce qui explique la grande variété des situations selon les lieux. L'étroitesse de la définition de religion légale a conduit la multitude d'activités, que l'anthropologie considère comme religieuses, à s'inventer une identité politiquement correcte (ainsi le qi gong pendant un temps) ou à se couler dans la conception d'un «patrimoine culturel immatériel », ainsi le culte 
de Confucius et même celui de Gengis-khan (p. 343). À Hong Kong et Macao, où le discours chrétien avait dominé le champ religieux jusqu'à ces derniers temps, le modèle, venu du continent, d'associations religieuses officiellement reconnues commence à se faire sentir. À Singapour, le mot d'ordre est celui d'une « Harmonie interreligieuse ». À Taiwan, l'essai de réinvention d'une " culture traditionnelle » a été abandonné après la levée de la loi martiale en 1987 au profit d'un libre pluralisme, qui profite notamment au mouvement du Yiguandao (chap. XII).

Il reste à évoquer les religions qui par leur origine «non-Han », se sont trouvées être un point de tension ou de relais au niveau international. Au premier chef vient le bouddhisme tibétain, qui, après une dure répression durant la Révolution culturelle, a connu, au début des années 1980 à la faveur de la décollectivisation, une reprise des constructions de temples et de monastères ainsi que des fondations extérieures au contrôle monastique (tel l'Institut Serta créé par Khenpo Jikphun en région Golok, au nord-ouest du Sichuan et ouvert aux femmes et aux Chinois Han) et l'élévation du Dalaï-lama, exilé, en chef religieux vénéré par l'ensemble de la population tibétaine et bien au-delà, en Mongolie-Intérieure et en République de Mongolie, en Chine Han, à Taiwan, en Occident, dans un mouvement religieux devenu mondial. Depuis les années 1990, les autorités chinoises veulent prendre en main la désignation des hauts réincarnés, et encore en nombre très limité (un cas emblématique a été la désignation du $\mathrm{XI}^{\mathrm{e}}$ Panchen-lama en 1995, p. 360-361). Les moines tibétains, soumis à une intense "éducation patriotique ", doivent faire face à un dilemme politique : soit reconstruire leur religion sous le contrôle de Pékin, soit participer à la lutte nationale pour l'indépendance du Tibet; et c'est cette seconde option qui est le plus souvent suivie jusqu'à nos jours. Les musulmans de langue chinoise en Chine continentale ont été victimes, comme les croyants de toute autre foi, de la folie de la Révolution culturelle (épisode particulièrement tragique du massacre de la petite ville de Shadian au Yunnan méridional en juillet 1975), mais la reconnaissance de leur religion comme le trait typique de leur appartenance " ethnique ", dite Hui, a permis une rapide renaissance des pratiques et de l'enseignement. Les instances officielles islamiques sont depuis 1950, comme à l'époque républicaine, tenues par des adhérents du mouvement Ikhwān (les "Frères »), antisoufi et nationaliste, mais le mouvement en réelle expansion depuis la fin du maösme est la Salafiyya, qui, en Chine, est non seulement antisoufie, anti-sinisation et fondamentaliste, mais aussi non-politique et antigouvernementale. Dans les villages de l'intérieur du pays, les adhésions dominantes sont soit, le plus couramment, celle dite gedimu (de l'arabe qadìm, "ancien "), c'est-à-dire l'observance de la traditionnelle sharī ${ }^{`} a$ ' telle qu'enseignée dans les mosquées locales, soit soufie (précisons que les confréries soufies ont dans l'ensemble, comme auparavant, leurs bases directoriales dans le Nord-Ouest du pays et le Yunnan). La tendance générale est à l'arabisation (ajoutons qu'auparavant le modèle culturel et la source littéraire principale venaient du monde persan) et à l'instrumentalisation 
de l'islam dans les rapports commerciaux et politiques de la RPC avec le monde islamique. Dans le Xinjiang turcophone, toute autre est la conjoncture car l'islam y est un instrument de résistance, parfois violente, à la domination Han (précisons que les Uyghurs rejettent les Hui à l'égal des Han, sans considération pour une prétendue fraternité religieuse) et doit se perpétuer plus ou moins clandestinement. Le gouvernement de Pékin, qui redoute un mouvement sécessionniste, a réussi, dans la foulée de l'attentat du 9 septembre 2001, à faire admettre devant l'opinion internationale que les Uyghurs sont tous des terroristes en puissance. Dans le cas du christianisme, les souffrances endurées par les croyants durant la Révolution culturelle ont renforcé leur foi et contribué à créer une mythologie du martyre fondateur.

Pour le catholicisme, dont la masse des fidèles s'accroît au même rythme que la population globale et qui ne présente aucun intérêt stratégique pour le gouvernement communiste, l'enjeu repose principalement dans les rapports officiels avec le Vatican (lequel reste un des rares États à continuer à reconnaître Taiwan comme République de Chine) et l'intégration de l'Église souterraine dans le courant accepté par le gouvernement. L'essor extraordinaire du christianisme chinois est, comme on l'a vu au chap. XI, le fait de l'aile la plus évangélique et nonconformiste du protestantisme, dans une clandestinité plus ou moins reconnue, notamment dans les réunions dans les maisons qui sont encouragées par les missionnaires américains et autres, et dans les communautés autochtones qui évoluent à la limite du sectarisme, condamné par le protestantisme académique. Sur le terrain, la situation est extrêmement complexe, car ni le catholicisme ni le protestantisme n'ont d'instance unifiant les diverses tendances (chap. XIII ; le cas des orthodoxes russes, des Juifs, des Bahā’i et des Mormons a été évoqué au chapitre XII).

La conclusion finale est déroutante et jette un éclairage inattendu sur l'ensemble de l'ouvrage. Nous tenons ici, certes, une remarquable histoire globale de la Chine et de la diaspora chinoise depuis quelque cent vingt ans, centrée sur les religions instituées et les phénomènes que l'anthropologie dénomme religieux ; tous les niveaux en sont explorés, dans toutes leurs nuances, et le rôle des femmes n'est pas oublié. L'exposé, particulièrement bien bâti et argumenté, que trahit la brièveté du présent résumé, se lit d'une traite et ne peut que convaincre le plus réticent des lecteurs que toute la vie sociale et politique chinoise est animée d'une façon ou d'une autre par des phénomènes religieux qui s'adaptent, à chaque époque, aux circonstances ponctuelles. C'est là le premier niveau de lecture qui s'impose dès les pages sur la Chine des Qing. Mais le propos va au-delà d'un simple cas d'école tout juste intéressant pour les sinologues et les sinomanes. À l'orée du Xxi e siècle, il est évident, à travers le monde, disent les coauteurs, que la perte de foi dans les idéologies sécularisées a favorisé la montée d'une religiosité réinventée. Or la Chine a fait preuve en ce domaine d'une inventivité unique en matière de création, de re-création et d'adaptation, de sorte que les 
comparatistes ne pourront éviter à l'avenir de se tourner vers les leçons que leur offre le laboratoire d'expériences qu'elle a constitué en un peu plus d'un siècle.

Une traduction française de l'ouvrage est annoncée. Le lecteur francophone aura plaisir à voir alors comment traduire des termes tels que "adscriptive society "; il sera reconnaissant aux auteurs si ceux-ci songent à donner systématiquement la transcription de tous les termes chinois cités dans le texte (elle manque, on ne sait pourquoi, au chapitre VII), et si, dans l'index, ils mentionnent, systématiquement aussi, la traduction des nombreuses expressions chinoises qu'on peine à retrouver in texto (ainsi qi gong n'est défini qu'à la quatrième occurrence, p. 120, comme " art de maîtriser son souffle"). Mais ce ne sont là que des broutilles : l'on songe, en refermant le livre, qu'il est l'illustration parfaite du dicton selon lequel "Le génie est de faire de l'inattendu avec du déjà-vu ».

L'ouvrage suivant, Hybrides chinois. La quête de tous les possibles, est formé de quatre conférences délivrées à la chaire du Louvre en mai 2011, et munies d'une copieuse annotation et de belles illustrations, par une historienne archéologue Danielle Elisseeff. Pourquoi donc classer sous la rubrique « Religions et politique aux $\mathrm{XX}^{\mathrm{e}}-\mathrm{XXI}^{\mathrm{e}}$ siècles » cette jolie promenade dans l'archéologie chinoise antique rapidement réglée en une centaine de pages? C'est que le sujet autour duquel virevolte la réflexion est le dragon, un fondement de la civilisation chinoise jusqu'à ce jour, et que l'argument central poursuit et illustre à la perfection celui de The Religious Question : les thèmes les plus antiques de l'art, dit la conférencière - en fait des thèmes religieux - peuvent disparaître quelque temps sous le coup d'événements contraires, mais ils réapparaissent immanquablement un jour ou l'autre, modifiés et adaptés à la nouvelle situation (l'on trouvera dans plusieurs des contributions de Religion et société en Chine ancienne et médiévale, recensé plus haut, l'arrière-plan socioreligieux des grandes trouvailles archéologiques).

La conférencière débusque les premiers traits d'un bestiaire sacré dès l'époque néolithique dans la grande plaine et dans le nord, aux $\mathrm{V}^{\mathrm{e}}-\mathrm{IV}^{\mathrm{e}}$ millénaires avant notre ère, notamment le dragon (sépulture de Hongshan, au Liaoning et en Mongolie-Intérieure) et le dragon, signe de l'Orient, associé au tigre, signe de l'Occident (dans une tombe de Xishuipo au Henan, relevant de la culture de Yangshao et fouillée en 1987-1988). Dans le grand ensemble palatial du début de l'âge du bronze, aménagé et occupé de 1700 à 1500 av. J.-C. (premiers règnes des Shang) découvert à Erlitou au Henan et fouillé en 2004, deux mille fines plaques de turquoise sont disposées en forme de dragon sur le corps d'un homme.

L'écriture, qui va nous permettre d'expliquer les rites, n'existe que plus tard et ne prend de l'importance qu'à la fin du $\mathrm{I}^{\mathrm{er}}$ millénaire avant notre ère. Au III ${ }^{\mathrm{e}}$ siècle av. J.-C., le dragon est alors indéniablement le véhicule de l'âme dans l'autre monde, mais un siècle plus tard, avec les Han, il est devenu l'allégorie du pouvoir impérial. Car, vers les environs de notre ère, les lettrés Han se sont efforcés de classer et de mettre en théorie les figures hybrides qui accompagnaient 
les morts un millénaire plus tôt, entre autres le dragon, un condensé invincible des forces de la nature. Les rapports avec les morts changent alors : on leur fournit désormais, dans leur demeure souterraine, un confort équivalent à celui qu'ils avaient connu de leur vivant. Dans les tombes des souverains, les statues de chevaux rappellent les mérites guerriers du défunt (tombe de Han Wudi, m. en 87 av. J.-C.). Au I ${ }^{\text {er }}$ siècle de notre ère, un lion est offert à la cour et son image dédoublée entre alors dans l'iconographie des êtres protecteurs : de nos jours deux lions de pierre ne signalent-ils pas l'entrée des banques et des hôtels (p. 85) ? Et il est aussi le gardien de la loi bouddhique. Car le bouddhisme, apparu depuis la fin du $\mathrm{I}^{\mathrm{er}}$ siècle de notre ère introduit en Chine de nouveaux hybrides dans le bestiaire sacré. Le dragon y gagne une association avec le serpent indien, le naga, qui confirme sa vieille alliance avec l'eau bienfaisante.

À l'époque Song, aux XI ${ }^{\mathrm{e}}-\mathrm{XII}{ }^{\mathrm{e}}$ siècles, le Livre des Mutations, le Yijing, entre dans le champ d'ardents débats sur la théorie et l'action et sur la corrélation du Ciel et de la Terre. C'est ainsi qu'il va pénétrer par l'entremise des écrits jésuites, à partir de la fin du XVII ${ }^{\mathrm{e}}$ siècle, dans la philosophie politique occidentale. Tandis que le petit peuple, pour vaincre ses anxiétés devant l'instabilité sociale (causée notamment par les invasions des peuples du nord du $\mathrm{X}^{\mathrm{e}}$ au XIII ${ }^{\mathrm{e}}$ siècle, puis de nouveau au XVII ${ }^{\mathrm{e}}$ ), installe tout autour de ses habitations des divinités, que même le régime maoïste ne pourra extirper. Lorsque les Ming donnent des signes de faiblesse vers la fin du XV et le début du XVI ${ }^{\mathrm{e}}$ siècle, une nouvelle iconographie se dessine, riche en épisodes miraculeux et propre à nourrir l'orgueil national : la biographie illustrée de Confucius. Les Qing, eux, popularisent les « huit signes de bon augure » que les souverains mongols des XIII ${ }^{\mathrm{e}}$-XIV ${ }^{\mathrm{e}}$ siècles avaient introduits $\mathrm{du}$ Tibet (vase, fleur de lotus, bannière, nœud sans fin, roue, ombrelle, poissons, conque).

Après la mort de Mao en 1976, les formes de pensée anciennes, voire archaïques, ressurgissent, ainsi les schémas du Yijing. Cet instructif survol se conclut sur la question : Confucius et Jésus peuvent-ils s'hybrider ?

S'il est un sujet où religion et politique sont mêlées, c'est bien celui des relations que la France a entretenues avec la Corée vers le milieu du XIX ${ }^{\mathrm{e}}$ siècle, ainsi que le montre avec pertinence le livre de Pierre-Emmanuel Roux, La croix, la baleine et le canon. Voici encore un auteur qui démonte des idées bien reçues pour éclairer les réalités, grâce au dépouillement d'archives et de documents en langues originales (y compris le coréen et le chinois) généralement peu consultés. Quelles sont donc ces idées reçues ? Entre 1836, année de l'arrivée à Séoul du premier missionnaire français, et 1876, année de la signature d'un premier traité franco-coréen, tous les dix ans - les « années des six » - il surviendrait un événement grave qui contraindrait la France à défendre avec ses canonnières le catholicisme naissant en Corée, car celui-ci était rejeté en raison du choc bien connu des civilisations, et à tenter de négocier par la force un accord diplomatique avec la dynastie coréenne régnante, les Chosŏn. 
Un premier mythe à démolir est celui de la prétendue "fermeture » du pays (ce concept, originellement forgé par un Européen puis traduit du hollandais en japonais par un interprète de Nagasaki, a été repris par les historiens japonais, et, à travers eux, par les Chinois et les Coréens, p. 43, no 1). De fait, intense était le commerce frontalier et maritime et constante et totale l'aide aux nombreux bateaux échoués sur les côtes coréennes, fussent-ils européens. Mais le récit des guerres de l'opium, parvenu avec retard à Séoul (en 1848 pour la guerre de 1839 et en 1859 pour la guerre de 1856) ancra le gouvernement coréen dans sa méfiance à l'égard des Occidentaux. Pour notre Monarchie de Juillet (1830-1848), la Corée devait être une porte d'entrée vers la Chine et, à partir de 1850, un point sur l'axe Cochinchine-Corée ; mais les expéditions maritimes du contre-amiral Jean-Baptiste Cécille en 1846, puis du capitaine de vaisseau Édouard Lapierre, naufragé en 1847, furent des échecs, qui eurent pour effet indirect une persécution du catholicisme (chap. I). Le thème de la défense des missionnaires, constamment avancé au milieu du XIX ${ }^{\mathrm{e}}$ siècle, cachait, en réalité, des intérêts purement mercantiles : la pêche à la baleine dans la mer du Japon. L'argument baleinier, soutenu à la fois par le Quai d'Orsay et la Marine, et en filigrane la recherche d'un "point d'appui " pour les baleiniers en Corée sont des questions peu étudiées qui font ici l'objet d'un chapitre très instructif (chap. II).

Cependant, dans la seconde moitié des années 1950, la Corée jouit d'une si mauvaise réputation que la diplomatie française s'en désintéresse pendant un temps, jusqu'à ce que des « interventionnistes » militants en relancent l'actualité, notamment l'ambitieux consul de France à Shanghaï, Louis-Charles de Montigny, et à Paris le fondateur des études japonaises en France, Léon de Rosny. La vengeance de l'exécution de neuf missionnaires français est le motif officiel de l'expédition militaire du contre-amiral Pierre-Gustave Roze en 1866, célèbre pour son échec et sa brutalité, mal ressentie par les intellectuels coréens jusqu'à nos jours encore. À cette époque, la vision d'une Corée pauvre et repoussante a laissé chez nous la place au mythe d'un marché fabuleux (chap. III et IV).

Les deux derniers chapitres révèlent le dessous des cartes de cette expédition de 1866. Les motifs profonds en sont, d'une part, stratégiques, à la suite de la menace que faisait peser sur les intérêts français l'avancée de l'empire russe jusqu'aux portes de la Corée (chap. V) ; et, d'autre part, vengeurs, à l'instigation de diplomates belliqueux et habiles à manier les exagérations et les mensonges (tel le chargé d'affaires Claude Bellonet, en particulier à propos du chiffre des victimes des mouvements antichrétiens), pour la prétendue défense des résidents français en Chine, au premier rang desquels les missionnaires. Mais finalement ni les menaces, ni les interventions militaires en Chine et en Corée ne purent garantir la sécurité des missionnaires et de leurs convertis, bien au contraire (chap. VI). En conclusion, il n'y a pas eu - de la monarchie de Juillet au Second Empire - de politique uniforme de la France vis-à-vis de la Corée, ni d'harmonie des vues entre Paris (qui temporisait) et les diplomates et officiers de marine 
en poste en Chine (qui poussaient aux actions guerrières aussi violentes que possible). Où était "la civilisation face aux barbares "? Du côté français, ou plus généralement européo-américain, ou du côté coréen, comme les autochtones attaqués chez eux en étaient convaincus ? Il est symptomatique que les bâtiments brûlés dans l'île de Kanghwa par Roze, lors de son expédition de 1866, aient été reconstitués à l'identique dans les années 1970 et que les manuscrits, saisis par lui dans les archives royales de l'île et découverts vers 1977 dans les dépôts de la Bibliothèque nationale de France, aient fait l'objet de tractations intergouvernementales serrées jusqu'à ce qu'un accord soit signé entre Paris et Séoul le 7 février 1911, prévoyant un prêt à la Corée renouvelable tous les cinq ans, soit, dans les faits, un retour définitif des précieux documents.

Ce récit très soigné, complété par une chronologie, vingt pages de notices biographiques, trente pages de bibliographie des sources et études en langues occidentales, en coréen et en chinois est centré sur l'expédition de 1866, au prix de quelques répétitions. Elle se lit comme un roman scandé d'extraits des lettres et rapports qui mettent en lumière, de réjouissante façon, les mensonges affichés et les motivations sous-jacentes des divers acteurs. C'est aussi un utile apport autant à l'histoire générale de la Chine et de l'Extrême-Orient au XIXe siècle qu'à l'histoire missionnaire; elle peut être également prise comme une saine leçon d'histoire diplomatique. 